\title{
The influence of knee position on ankle dorsiflexion - a biometric study
}

\author{
Sebastian F Baumbach*, Mareen Brumann, Jakob Binder, Wolf Mutschler, Markus Regauer and Hans Polzer
}

\begin{abstract}
Background: Musculus gastrocnemius tightness (MGT) can be diagnosed by comparing ankle dorsiflexion (ADF) with the knee extended and flexed. Although various measurement techniques exist, the degree of knee flexion needed to eliminate the effect of the gastrocnemius on ADF is still unknown. The aim of this study was to identify the minimal degree of knee flexion required to eliminate the restricting effect of the musculus gastrocnemius on ADF.

Methods: Bilateral ADF of 20 asymptomatic volunteers aged $18-40$ years ( $50 \%$ female) was assessed prospectively at six different degrees of knee flexion $\left(0^{\circ}, 20^{\circ}, 30^{\circ}, 45^{\circ}, 60^{\circ}, 75^{\circ}\right.$, Lunge). Tests were performed following a standardized protocol, non weightbearing and weightbearing, by two observers. Statistics comprised of descriptive statistics, t-tests, repeated measurement ANOVA and ICC.
\end{abstract}

Results: 20 individuals with a mean age of $27 \pm 4$ years were tested. No significant side to side differences were observed. The average ADF [95\% confidence interval] for non weightbearing was $4^{\circ}\left[1^{\circ}-8^{\circ}\right]$ with the knee extended and $20^{\circ}\left[16^{\circ}-24^{\circ}\right]$ for the knee $75^{\circ}$ flexed. Mean weightbearing ADF was $25^{\circ}\left[22^{\circ}-28^{\circ}\right]$ for the knee extended and $39^{\circ}\left[36^{\circ}-42^{\circ}\right]$ for the knee $75^{\circ}$ flexed. The mean differences between $20^{\circ}$ knee flexion and full extension were $15^{\circ}\left[12^{\circ}-18^{\circ}\right]$ non weightbearing and $13^{\circ}\left[11^{\circ}-16^{\circ}\right]$ weightbearing. Significant differences of ADF were only found between full extension and $20^{\circ}$ of knee flexion. Further knee flexion did not increase ADF.

Conclusion: Knee flexion of $20^{\circ}$ fully eliminates the ADF restraining effect of the gastrocnemius. This knowledge is essential to design a standardized clinical examination assessing MGT.

Keywords: Musculus gastrocnemius tightness, Knee flexion, Range of motion, Ankle joint

\section{Background}

Various pathologies affecting the lower extremity, including plantar heel pain [1-3], metatarsalgia [4,5], stress fractures of the foot, and Achilles tendionpathy [6] are associated with limited ankle dorsiflexion (ADF). During gait reduced ADF results in an increase of forefoot pressure, which might be responsible for the above outlined pathologies [7-9]. Studies were able show, that increasing ADF in these patients leads to a reduction of the symptoms [10-13]. ADF can be impaired due to osseous, ligmentous, neurologic or muscular restrains, with musculus gastrocnemius tightness (MGT) being the most common cause $[1,14]$.

\footnotetext{
* Correspondence: sebastian.baumbach@med.uni-muenchen.de Department of Trauma Surgery - Campus Innenstadt, Munich University Hospital, Nussbaumstr. 20, 80336 Munich, Germany
}

The musculus gastrocnemius has an influence on ADF because it bridges the knee and ankle joint. Under physiological conditions the gastrocnemius is under full tension when the knee is extended, as the muscle's origin is furthest from its insertion. ADF is then restrained by the muscle's tension. On the contrary, knee flexion increases ADF, as the muscle's origin and insertion are approximated. Further $\mathrm{ADF}$ is then limited by other structures of the ankle joint (Figure 1). In symptomatic patients the first assessment should therefore evaluate ADF with the knee fully extended. In case of impaired ADF one should then identify whether ADF can be increased by knee flexion. Patients with MGT demonstrate a reduced ADF with the knee fully extended, but ADF can be increased by knee flexion [2]. Identification of isolated MGT is essential for both physiotherapists and physicians, as it can be treated by stretching or endoscopic musculus gastrocnemius recession. If ADF 


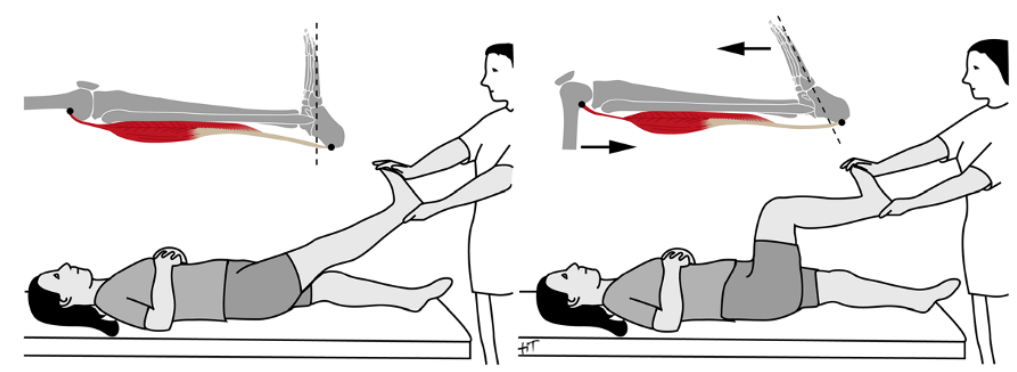

Figure 1 Schematic illustration of the anatomical and testing principles of the relation between ankle dorsiflexion and the knee position.

cannot be increased by knee flexion the gastrocnemius is not responsible for the impaired ADF.

Consequently, any clinical test investigating MGT compares ADF with the knee extended to ADF with the knee flexed. This procedure was first described in 1923 by Nils Otto Silfverskiöld, an Swedish orthopedic surgeon [15].

In the literature, numerous tests are described to assess ADF. These tests can be categorized into non weightbearing $[3,16]$, weightbearing $[17,18]$, and instrumented $[19,20]$. In everyday practice non weightbearing measurements are most commonly performed [21]. Nevertheless, evidence suggests a higher intra- and interrater reliability for weightbearing compared to non weightbearing measurements [17,18,21-23]. Furthermore, maximum ADF significantly differs from weightbearing to non weightbearing [23].

Independent of the test applied, no standard has been defined for the degree of knee flexion needed to eliminate the effect of the musculus gastrocnemius on ADF. Most studies conducting non weightbearing measurements applied a knee flexion of $90^{\circ}[3,7,16]$. On the contrary, most weightbearing measurements do not control for knee flexion [16,23]. Furthermore, weightbearing measurements with $90^{\circ}$ knee flexion are not feasible. Consequently, it is of upmost importance to identify the minimal required degree of knee flexion to eliminate the effect of the musculus gastrocnemius on ADF.
To our best knowledge the degree of knee flexion needed to eliminate the restraining effect of the gastrocnemius on ADF is unknown. Therefore, the aim of this study was to identify the influence of varying amounts of knee joint flexion on ADF.

\section{Methods}

\section{Study design and population}

The study was approved by the local ethics committee of the University of Munich (\# 007-14). Both ankles of 20 healthy individuals, aged $18-40$ years, $50 \%$ female, were tested according to a standardized protocol, following screening and informed consent. The inclusion and exclusion criteria are presented in Table 1.

\section{Measurement procedure}

ADF measurements were conducted both weightbearing and non weightbearing at different degrees of knee flexion and in a lunge position, following a standardized protocol. Each measurement was performed by two investigators (SFB, HP), blinded to each other's results. A standard goniometer (MDF Instruments USA, Inc. Malibu, CA, USA) with $2^{\circ}$ increments and $20 \mathrm{~cm}$ length was used. Anatomical measurement landmarks were the long axis of the fibula and the fifth metatarsal bone [16,24-27], which were marked prior to testing [24,28]. A functional brace (Medi M4, Medi GmbH \& Co. KG, Bayreuth, Germany),

Table 1 Inclusion and exclusion criteria

\begin{tabular}{ll}
\hline Inclusion criteria & Exclusion criteria \\
\hline Age: 18 to 40 years & Prior injuries to the knee, ankle or foot \\
Informed consent & Knee, ankle or foot pain within the last 2 years \\
Subjects can read and understand German & History of ankle trauma or surgery \\
& Impaired knee range of motion \\
& Conditions/systematic diseases possibly affecting the neuromuscular or musculoskeletal system \\
& Pregnancy [anamnestic] \\
& Cardiovascular diseases including thrombosis \\
& Regular medication excluding contraceptives \\
& Subject is unable to give informed consent \\
\hline
\end{tabular}


which fixes the knee in various angles, was used to control the knee position. ADF was assessed at the following degrees of knee flexion: full extension, $20^{\circ}, 30^{\circ}, 45^{\circ}$, $60^{\circ}$ and $75^{\circ}$. The order of examiners and knee flexion (full extension to $75^{\circ}$ vs. $75^{\circ}$ to full extension) were altered between the subjects. The subjects rested for 30 seconds between measurements.

Non weightbearing ADF measurements were taken with the individuals in supine position. One investigator applied maximum ADF with the foot in subtalar neutral position, while the other performed the measurement (Figure 2A; the shown persons gave informed consent for publishing their image).

For weightbearing ADF measurements, the subject was asked to stand in a lunge position with the back leg being the one measured. The second toe and heel were centered over a line perpendicular to the wall. Patients were allowed to stabilize their stance by holding onto the wall. For the full dorsiflexion measurements, the patient was then asked to lean forward just before heel lift off. One examiner assured maximum knee extension. For the other measurements, the subject was asked to flex their knee until fully restrained by the functional brace and then move their knee forward above the line just before heel lift off. The other examiner assured subtalar neutral position (Figure 2B).

The final test performed was the Lunge test. The subject was asked to do a lunge with the back leg flexed and squad with the rear leg until just before the heel lifts off the ground. The second toe and heel were again centered over a line perpendicular to the wall. Patients were allowed to stabilize their stance by holding onto the wall. Subtalar neutral position was monitored (Figure 2C). The references used for all weightbearing measurements were the long axis of fibula and the ground.

\section{Outcome variables and statistics}

Data assessed were standard demographics, level of sports, dominant leg (defined as leg used to kick a ball), and maximum ADF. Values are presented as mean values \pm standard deviations or the $95 \%$ confidence intervals $(\mathrm{CI})$.

A sample size calculation could not be performed due to missing preliminary data. The sample size was chosen based on previous studies on MGT [2,18,21]. MannWhitney-U-Tests were used to compare gender-, side-, and non-/weightbearing differences. A Bonferroni correction was used to account for multiple testing $(\mathrm{p}<0.004)$. A repeated measurement ANOVA was conducted to assess significant differences for ADF between the different degrees of knee flexion. Test reliability was assessed by interrater reliability using the interclass correlation coefficient (ICC). Interrater reliability defines the consistency of a measure taken by two examiners. ICC values range from 0 to 1 , with 1 being perfect agreement. In general, an ICC greater than 0.7 is considered an acceptable level of reliability for clinical measures $[29,30]$. If not stated differently the values stated are the mean values of both investigators. Statistics were computed using SPSS Vs. 21 (IBM Company).

\section{Results}

\section{Participant characteristics}

Twenty healthy individuals (50\% female) with a mean age of $27.1 \pm 3.9$ years (height: $175.0 \pm 9.8 \mathrm{~cm}$; weight: $68.7 \pm 10.9 \mathrm{~kg}$ ) were examined. The dominant foot, defined as the foot used to kick a ball was in all but one case the right foot. All but one participant (no sport) indicated that they participate in sporting activities 2-3 times per week. Statistical assumptions for normal distribution were not met (D'Agostino and Pearson Test). Table 2 shows the descriptive statistics for $\mathrm{ADF}$ for each degree of knee flexion separately.

\section{Reproducability analysis}

The interrater ICC ranged from 0.971 to 0.988 for non weightbearing, and from 0.961 to 0.992 for weightbearing measurements.

\section{Inferential analysis}

No significant gender differences could be found for any measurement. A Mann-Whitney-U-Test revealed

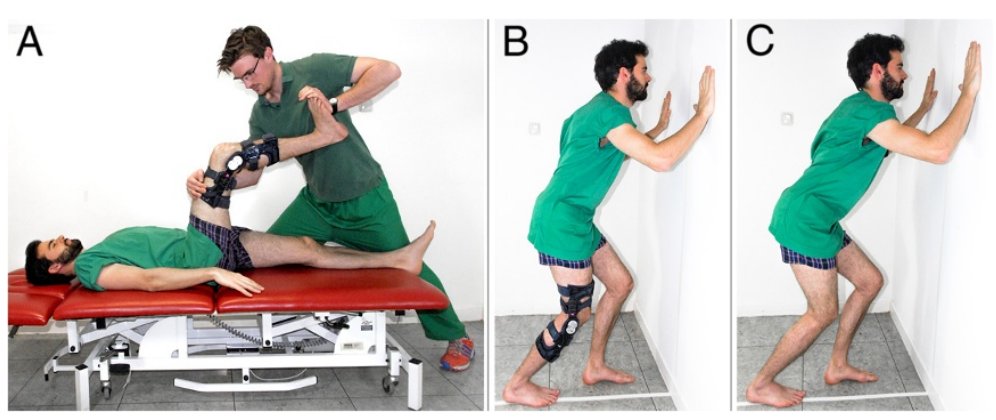

Figure 2 Exemplary test procedure. A) Non weightbearing measurement; B) Weightbearing measurement; C) Lunge test; The image was taken by the authors and the shown persons gave informed consent for publishing their image. 
Table 2 Degrees of ankle dorsiflexion at various degrees of knee flexion

\begin{tabular}{lccccccc}
\hline Knee flexion & Full Ext. & $\mathbf{2 0}^{\circ}$ & $\mathbf{3 0}^{\circ}$ & $\mathbf{4 5}^{\circ}$ & $\mathbf{6 0}^{\circ}$ & $\mathbf{7 5}^{\circ}$ & Lunge $^{\circ}$ \\
\hline NWB right & $4 \pm 8$ & $20 \pm 9$ & $21 \pm 9$ & $21 \pm 9$ & $21 \pm 9$ & $21 \pm 9$ & $39 \pm 7$ \\
WB right & $25 \pm 7$ & $39 \pm 7$ & $39 \pm 7$ & $39 \pm 7$ & $39 \pm 7$ & $40 \pm 7$ \\
NWB left & $4 \pm 7$ & $18 \pm 7$ & $19 \pm 9$ & $20 \pm 10$ & $20 \pm 10$ & $20 \pm 9$ \\
WB left & $24 \pm 6$ & $37 \pm 6$ & $38 \pm 7$ & $38 \pm 6$ & $38 \pm 7$ & $38 \pm 7$ & $39 \pm 7$ \\
\hline
\end{tabular}

Values given as mean \pm standard deviation; ${ }^{\circ}$ : degrees; NWB: non weightbearing; WB: weightbearing; Full Ext.: Full extension.

no significant differences between the right and left leg for neither non weightbearing nor weightbearing measurements. Pooled mean ADF (mean values of the right and left ankle) for the extended knee non weightbearing were $4^{\circ} \pm 7^{\circ}\left(95 \% \mathrm{CI}: 1^{\circ}-8^{\circ}\right.$; range: $\left.11^{\circ}-23^{\circ}\right)$ and weightbearing $25^{\circ} \pm 6^{\circ}\left(95 \% \mathrm{CI}: 22^{\circ}-28^{\circ}\right.$; range: $\left.16^{\circ}-40^{\circ}\right)$. For the knee $75^{\circ}$ flexed non weightbearing values were $20 \pm$ $9^{\circ}\left(95 \% \mathrm{CI}: 16^{\circ}-24^{\circ}\right.$; range: $\left.9^{\circ}-49^{\circ}\right)$ and weightbearing $39^{\circ} \pm 7^{\circ}\left(95 \%\right.$ CI: $36^{\circ}-42^{\circ}$; range: $\left.31^{\circ}-58^{\circ}\right)$. The pooled data for non weightbearing and weightbearing ADF measurements and the differences between each step of knee flexion (delta) are presented in Figure 3. Weightbearing measurements were significantly greater than non weightbearing values (Mann- Whitney-U-Test, $\mathrm{p}<0.001)$.

A repeated measurement ANOVA revealed significant differences $(\mathrm{p}<0.001)$ between $0^{\circ}$ and all other degrees of knee flexion $\left(20^{\circ}, 30^{\circ}, 45^{\circ}, 60^{\circ}, 75^{\circ}\right.$, Lunge). No significant differences for ADF could be observed when comparing $20^{\circ}$ to $75^{\circ}$ of knee flexion including the Lunge test. Consequently, knee flexion beyond $20^{\circ}$ could not further increase ADF.

The difference for ADF (delta) between $20^{\circ}$ knee flexion and full extension was $15^{\circ} \pm 6^{\circ}$ [CI: $12^{\circ}-18^{\circ}$ for non weightbearing and $13^{\circ} \pm 5^{\circ}\left[\mathrm{CI}: 11^{\circ}-16^{\circ}\right]$ for weightbearing ADF, which did not differ significantly. Starting

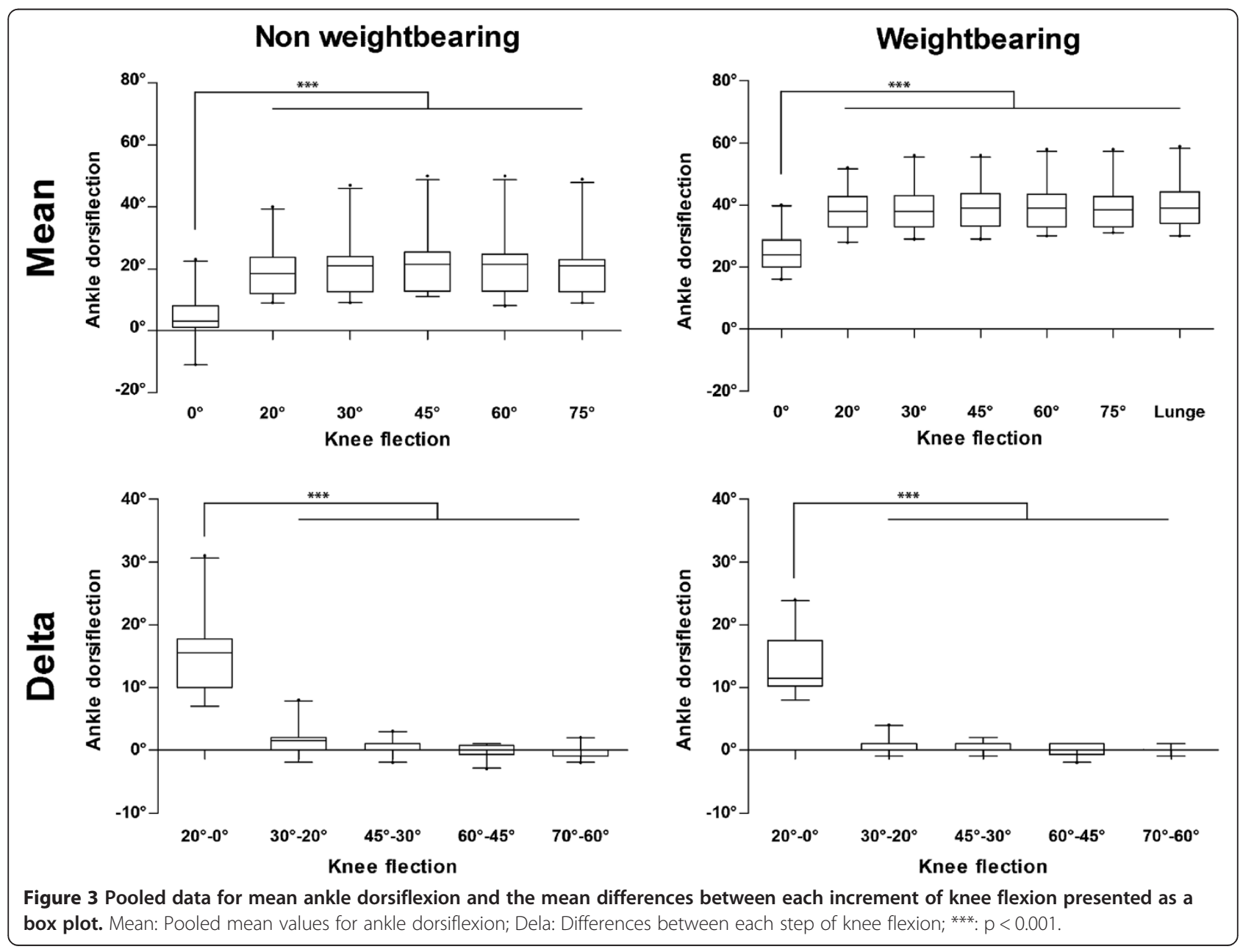


from $20^{\circ}$ knee flexion the maximum delta value observed was $1^{\circ}\left[\mathrm{CI}: 0^{\circ}-3^{\circ}\right]$ for non weightbearing and $1^{\circ}\left[\mathrm{CI}: 0^{\circ}-1^{\circ}\right]$ for weightbearing.

\section{Discussion}

Musculus gastrocnemius tightness (MGT) is the most common cause for impaired dorsiflexion of the ankle joint $[1,14]$. It is held responsible for various pathologies affecting the lower extremity [4-6]. Identification of this cause by the treating physiotherapist or physician is extremely important as it can be easily treated by gastrocnemius lengthening either through physiotherapy or surgery. Various tests try to assess MGT by comparing ADF with the knee extended and flexed $[3,18,20]$. None of these tests has identified the degree of knee flexion needed to eliminate the effect of the gastrocnemius on ADF. Our study is the first to investigate the influence of the knee's position on ADF. By $20^{\circ}$ of knee flexion, the ADF restraining effect of a tight gastrocnemius on was already eliminated such that further knee flexion provided no additional ADF.

In the literature, different anatomical landmarks are described to assess the range of motion of the ankle joint. The landmarks most frequently used are the long axis of the fibula and either the plantar surface of the foot [21,31-33] or the axis of the fifth metatarsal bone [16,24-27,34-36]. During pretests, we found the accurate identification of the plantar aspect of the foot to be highly dependent on the hand position of the examiner. Therefore, we chose the long axis of the fibula and the fifth metatarsal bone as references for non weightbearing measurements. For weightbearing tests, the floor clearly defined the $\mathrm{x}$-axis, but the long axis of the fibula was hard to be identified due to the prominence of the peroneal tendons while standing. We therefore decided to mark the long axis of the fibula and the fifth metatarsal bone prior to testing $[24,28]$. This procedure was also employed by Astroem and Arvidson [28] who conducted range of motion measurements to the foot in 121 healthy subjects. One has to keep in mind, that this might have a positive impact on the interrater reliability. In our study the interrater ICC values ranged from 0.961 to 0.992 . These values are excellent, especially when compared to other non weightbearing measurements, with values ranging between 0.29 [36] and 0.81 [37]. Comparably good values have only been reported for weightbearing ADF measurements [17,38]. Our goal was to clearly define the degree of knee flexion needed to eliminate the effect of the musculus gastrocnemius on ankle dorsiflexion. Consequently, it was crucial to use a reliable measurement technique. The herein observed interclass correlation coefficient argues for our standardized measurement protocol.

We observed a great variation of ADF between the subjects with values ranging for the extended knee from $-11^{\circ}$ to $23^{\circ}$ non weightbearing and from $16^{\circ}$ to $40^{\circ}$ weightbearing. For the knee $75^{\circ}$ flexed values ranged from $9^{\circ}$ to $49^{\circ}$ non weightbearing and from $31^{\circ}$ to $58^{\circ}$ weightbearing. Comparable variations for ADF have been reported in the literature. Non weightbearing values range from $-2^{\circ} \pm 5^{\circ}$ [16] to $20^{\circ} \pm 5^{\circ}$ [3] for the knee extended and from $12^{\circ} \pm 6^{\circ}$ [16] to $25^{\circ} \pm 5^{\circ}$ [3] for the knee flexed. Furthermore, we could observe significant differences between weightbearing and non weightbearing ADF measurements. This goes well in line with the observation of other authors $[2,16,18,21,23,28]$. Although there is less data available for weightbearing measurements, reported values range from $21^{\circ} \pm 7^{\circ}$ [21] to $39^{\circ} \pm 5^{\circ}$ [18] for the knee extended and from $33^{\circ} \pm 7^{\circ}$ [16] to $50^{\circ} \pm 6^{\circ}$ [23] for the knee flexed. The great range of ADF values might be due to the heterogeneity of the measurement procedures and the different landmarks used. Another pitfall we observed during the pretests was that minimal knee flexion had a profound impact on the ADF when measuring the ADF with the knee extended. Consequently, for our testing procedure one observer ensured full extension of the knee during measurements. Moreover, the great range of ADF might simply reflect the constitutional variation within the population.

Limitations that should be discussed are adjacent joint movements affecting ADF and the measurement device used. ADF is not limited to the tibiotalar joint, but also occurs partially in the subtalar and midtarsal joints. We tried to account for this problem by maintaining the foot in a subtalar neutral position, as recommended by previous authors [39-42]. Second, the goniometer used has $2^{\circ}$ increments. Although other devices might be more accurate, the goniometer has to be considered the clinical gold standard [30]. With respect to the high ICC observed we believe this tool to be sufficiently accurate. Furthermore, because 20 degrees was the smallest knee flexion angle tested after full knee extension, it might be possible that even less knee flexion is already sufficient.. Nevertheless, we believe that every patient is capable to perform a Lunge test with $20^{\circ}$ of knee flexion.

As stated above decreased ADF is held responsible for a variety of disorders. Up to date, there is neither a consensus on the degree of ADF considered pathological, nor whether tests should be conducted non weightbearing or weightbearing. The latter though has a pronounce impact on the degree of ADF measured. Weightbearing measurements have several advantages. First, they can be conducted by a single investigator. Second, they are independent of the torque applied by the observer, being a possible source of bias, and in addition more closely reflect the physiological torque during gait. Third, they have been shown to be more reliable [17,18,21-23]. Considering these aspects weightbearing tests should become the clinical standard. Nevertheless, most patients cannot 
conduct weightbearing tests with the knee $90^{\circ}$ flexed, as conducted in non weightbearing measurements $[3,7,16]$. Consequently, it is of upmost importance to identify the minimal degree of knee flexion needed to eliminate the ADF restraining effect of the musculus gastrocnemius. This study is the first to investigate this problem. We were able to clearly demonstrate that already $20^{\circ}$ of knee flexion sufficiently eliminates the effect of the musculus gastrocnemius on ADF.

\section{Conclusion}

When assessing MGT the degree of knee flexion needed to eliminate the restraining effect of the musculus gastrocnemius on ADF was unknown. We were able to demonstrate that already $20^{\circ}$ of knee flexion fully eliminates the restraining effect of the musculus gastrocnemius on ADF, both non weightbearing and weightbearing. Our results build the bases to define a standardized clinical examination for musculus gastrocnemius tightness.

\section{Abbreviations}

ADF: Ankle dorsiflexion; MGT: Musculus gastrocnemius tightness; \%: Percent; ICC: Interclass correlation coefficient; ${ }^{\circ}$ : Degrees; \#: Number; Cm: Centimeters; Kg: Kilograms.

\section{Competing interests}

The authors declare that they have no competing interests.

\section{Authors' contributions}

SFB was involved in developing the study design, data acquisition and analysis and prepared the manuscript. MB reviewed the test setup and participated in the data acquisition. JB was responsible for subject acquisition, time management and test implementation. WM was involved in developing the study design, was an essential part of data interpretation. MR helped design the test setup acquire the data and prepared parts of the manuscript. HP had the study idea, was an essential party of data acquisition and interpretation and prepared wide parts of the manuscript. All authors read and approved the final manuscript.

\section{Acknowledgements}

We thank Mrs. Hella Thun who designed all figures. Furthermore, we thank Medi GmbH \& Co. KG (Bayreuth, Germany) for the provision of the Medi M4 orthoses. Moreover, we thank Mr. Dipl.-Ing. (FH), M.Sc. M. Saller for his statistical support.

\section{Received: 24 February 2014 Accepted: 15 July 2014}

Published: 23 July 2014

\section{References}

1. Patel A, DiGiovanni B: Association between plantar fasciitis and isolated contracture of the gastrocnemius. Foot Ankle Int 2011, 32:5-8.

2. DiGiovanni CW, Kuo R, Tejwani N, Price R, Hansen ST, Cziernecki J, Sangeorzan BJ: Isolated gastrocnemius tightness. J Bone Joint Surg Am 2002, 84-A:962-970.

3. Bolívar YA, Munuera PV, Padillo JP: Relationship between tightness of the posterior muscles of the lower limb and plantar fasciitis. Foot Ankle Int 2013, 34:42-48.

4. Irving DB, Cook JL, Menz HB: Factors associated with chronic plantar heel pain: a systematic review. J Sci Med Sport 2006, 9:11-22. discussion 23-14.

5. Crawford F, Thomson C: Interventions for treating plantar heel pain. Cochrane Database Syst Rev 2003, 3:CD000416.

6. Wilder RP, Sethi S: Overuse injuries: tendinopathies, stress fractures, compartment syndrome, and shin splints. Clin Sports Med 2004, 23:55-81. vi.
7. You J-Y, Lee H-M, Luo H-J, Leu C-C, Cheng P-G, Wu S-K: Gastrocnemius tightness on joint angle and work of lower extremity during gait. Clin Biomech (Bristol, Avon) 2009, 24:744-750.

8. Aronow MS, Diaz-Doran V, Sullivan RJ, Adams DJ: The effect of triceps surae contracture force on plantar foot pressure distribution. Foot Ankle Int 2006, 27:43-52.

9. Chimera NJ, Castro M, Davis I, Manal K: The effect of isolated gastrocnemius contracture and gastrocnemius recession on lower extremity kinematics and kinetics during stance. Clin Biomech (Bristol, Avon) 2012, 27:917-923.

10. Duthon VB, Lübbeke A, Duc SR, Stern R, Assal M: Noninsertional Achilles tendinopathy treated with gastrocnemius lengthening. Foot Ankle Int 2011, 32:375-379.

11. Nishimoto GS, Attinger CE, Cooper PS: Lengthening the Achilles tendon for the treatment of diabetic plantar forefoot ulceration. Surg Clin North Am 2003, 83:707-726.

12. Barske HL, DiGiovanni BF, Douglass M, Nawoczenski DA: Current concepts review: isolated gastrocnemius contracture and gastrocnemius recession. Foot Ankle Int 2012, 33:915-921.

13. Gurdezi S, Kohls-Gatzoulis J, Solan MC: Results of proximal medial gastrocnemius release for Achilles tendinopathy. Foot Ankle Int 2013, 34:1364-1369.

14. Hill RS: Ankle equinus. Prevalence and linkage to common foot pathology. J Am Podiatr Med Assoc 1995, 85:295-300.

15. Silfverskiold N: Reduction of the uncrossed two-joints muscles of the leg to one-joint muscles in spastic conditions. Acta Chir Scand 1923, 56:315-330

16. Krause DA, Cloud BA, Forster LA, Schrank JA, Hollman JH: Measurement of ankle dorsiflexion: a comparison of active and passive techniques in multiple positions. J Sport Rehabil 2011, 20:333-344.

17. Bennell KL, Talbot RC, Wajswelner H, Techovanich W, Kelly DH, Hall AJ: Intra-rater and inter-rater reliability of a weight-bearing lunge measure of ankle dorsiflexion. Aust J Physiother 1998, 44:175-180.

18. Munteanu SE, Strawhorn AB, Landorf KB, Bird AR, Murley GS: A weightbearing technique for the measurement of ankle joint dorsiflexion with the knee extended is reliable. J Sci Med Sport 2009, 12:54-59.

19. Wilken J, Rao S, Estin M, Saltzman CL, Yack HJ: A new device for assessing ankle dorsiflexion motion: reliability and validity. J Orthop Sports Phys Ther 2011, 41:274-280.

20. Gatt A, Chockalingam N: Validity and reliability of a new ankle dorsiflexion measurement device. Prosthetics Orthot Int 2013, 37:289-297.

21. Baggett BD, Young G: Ankle joint dorsiflexion. Establishment of a normal range. J Am Podiatr Med Assoc 1993, 83:251-254.

22. Menz HB, Tiedemann A, Kwan MM-S, Latt MD, Sherrington C, Lord SR Reliability of clinical tests of foot and ankle characteristics in older people. J Am Podiatr Med Assoc 2003, 93:380-387.

23. Rabin A, Kozol Z: Weightbearing and nonweightbearing ankle dorsiflexion range of motion: are we measuring the same thing? J Am Podiatr Med Assoc 2012, 12:406-411.

24. Worrell TW, McCullough M, Pfeiffer A: Effect of foot position on gastrocnemius/soleus stretching in subjects with normal flexibility. J Orthop Sports Phys Ther 1994, 19:352-356.

25. Norkin CC, White DJ: Measurement of Joint Motion: A Guide to Goniometry. 3rd edition. Philadelphia: FA Davis; 2003.

26. Jonson SR, Gross MT: Intraexaminer reliability, interexaminer reliability, and mean values for nine lower extremity skeletal measures in healthy naval midshipmen. J Orthop Sports Phys Ther 1997, 25:253-263.

27. Seiger C, Draper DO: Use of pulsed shortwave diathermy and joint mobilization to increase ankle range of motion in the presence of surgical implanted metal: a case series. J Orthop Sports Phys Ther 2006, 36:669-677.

28. Aström M, Arvidson T: Alignment and joint motion in the normal foot. J Orthop Sports Phys Ther 1995, 22:216-222.

29. Lahey MA, Downey RG, Saal FE: Intraclass correlations: there is more than meets the eye. Psychol Bull 1983, 93:586-595.

30. Martin RL, McPoil TG: Reliability of ankle goniometric measurements: a literature review. J Am Podiatr Med Assoc 2005, 95:564-572.

31. Pandya S, Florence JM, King WM, Robison JD, Oxman M, Province MA: Reliability of goniometric measurements in patients with Duchenne muscular dystrophy. Phys Ther 1985, 65:1339-1342. 
32. Kim PJ, Peace R, Mieras J, Thoms T, Freeman D, Page J: Interrater and intrarater reliability in the measurement of ankle joint dorsiflexion is independent of examiner experience and technique used. $J$ Am Podiatr Med Assoc 2011, 101:407-414.

33. Youdas JW, Krause DA, Egan KS, Therneau TM, Laskowski ER

The effect of static stretching of the calf muscle-tendon unit on active ankle dorsiflexion range of motion. J Orthop Sports Phys Ther 2003, 33:408-417.

34. Fosang AL, Galea MP, McCoy AT, Reddihough DS, Story I: Measures of muscle and joint performance in the lower limb of children with cerebral palsy. Dev Med Child Neurol 2003, 45:664-670.

35. Kilgour G, McNair P, Stott NS: Intrarater reliability of lower limb sagittal range-of-motion measures in children with spastic diplegia. Dev Med Child Neurol 2003, 45:391-399.

36. Van Gheluwe B, Kirby KA, Roosen P, Phillips RD: Reliability and accuracy of biomechanical measurements of the lower extremities. J Am Podiatr Med Assoc 2002, 92:317-326.

37. Diamond JE, Mueller MJ, Delitto A, Sinacore DR: Reliability of a diabetic foot evaluation. Phys Ther 1989, 69:797-802

38. Venturini C, Ituassu NT, Teixeira LM, Deus CVOE: Intrarater and interrater reliability of two methods for measuring the active range of motion for ankle dorsiflexion in healthy subjects. Rev bras fisioter 2006, 10:407-411.

39. Tiberio D: Evaluation of functional ankle dorsiflexion using subtalar neutral position. Clin Rep Phys Ther 1987, 67:955-957.

40. Tiberio D, Bohannon RW, Zito MA: Effect of subtalar joint position on the measurement of maximum ankle dorsiflexic. Clin Biomech (Bristol, Avon) 1989, 4:189-191.

41. Bohannon RW, Tiberio D, Waters G: Motion measured from forefoot and hindfoot landmarks during passive ankle dorsiflexion range of motion. J Orthop Sports Phys Ther 1991, 13:20-22.

42. Johanson M, Baer J, Hovermale H, Phouthavong P: Subtalar joint position during gastrocnemius stretching and ankle dorsiflexion range of motion. J Athl Train 2008, 43:172-178.

doi:10.1186/1471-2474-15-246

Cite this article as: Baumbach et al:: The influence of knee position on ankle dorsiflexion - a biometric study. BMC Musculoskeletal Disorders 2014 15:246.

\section{Submit your next manuscript to BioMed Central and take full advantage of:}

- Convenient online submission

- Thorough peer review

- No space constraints or color figure charges

- Immediate publication on acceptance

- Inclusion in PubMed, CAS, Scopus and Google Scholar

- Research which is freely available for redistribution 